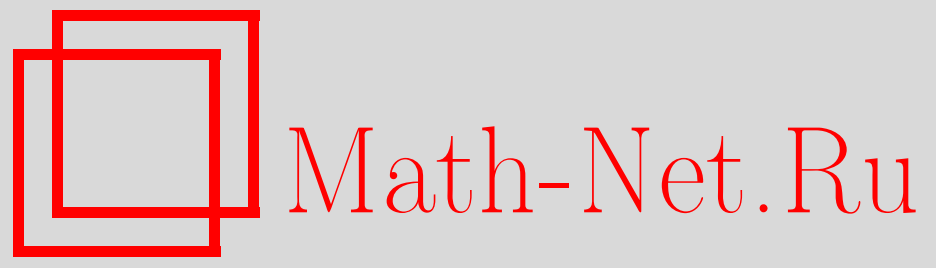

A. M. Kagan, C. R. Rao, On estimation of a location parameter in presence of an ancillary component, Теория вероятн. и ее примен., 2005, том 50, выпуск 1, 172-176

DOI: https://doi.org/10.4213/tvp166

Использование Общероссийского математического портала Math-Net.Ru подразумевает, что вы прочитали и согласны с пользовательским соглашением

http://www . mathnet.ru/rus/agreement

Параметры загрузки:

IP: 3.89 .197 .203

26 апреля 2023 г., 13:52:59

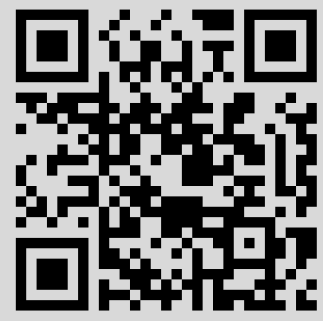


4. Chow Y.S., Teicher H. Probability Theory: Independence, Interchangeability, Martingales. New York: Springer-Verlag, 1997, 488 p.

5. Dellacherie C., Meyer P.A. Probabilities and Potential. B. Theory of Martingales. Amsterdam: North-Holland, 1982, $463 \mathrm{p}$.

6. Gundy R. F. On the class $L \log L$, martingales, and singular integrals. - Studia Math., 1969 , v. 33, p. 109-118.

7. Long R. Martingale Spaces and Inequalities. Beijing/Braunschweig: Peking Univ. Press/Vieweg, 1993, $346 \mathrm{p}$.

8. Neveu J. Discrete-Parameter Martingales. Amsterdam: North-Holland, 1975, 236 p.

Поступила в редакцию

10.XII. 2003

(C) $2005 \mathrm{r}$

KAGAN A.*, RAO C. R.**

\section{ON ESTIMATION OF A LOCATION PARAMETER IN PRESENCE OF AN ANCILLARY COMPONENT ${ }^{1)}$}

Если $(X, Y)$ есть наблюдение случайного вектора с функцией распределения $F(x-\theta, y), \sigma^{2}=\mathbf{D} X, \rho=\operatorname{corr}(X, Y)$ и $I-$ информация Фишера о параметре $\theta$ в $(X, Y)$, то $I \geqslant\left\{\sigma^{2}\left(1-\rho^{2}\right)\right\}^{-1}$.

Равенство достигается при выполнении условий, тесно связанных с условиями линейности оценки Питмэна для $\theta$ по выборке из совокупности $F(x-\theta, y)$. Эти утвержения обобщают результаты, полученные ранее для случая, когда наблюдается только компонента $X$.

Ключевые слова и фразы: информация Фишера, оценка Питмэна.

1. Introduction. Let $\left(x_{1}, \ldots, x_{n}\right)$ be a sample of size $n \geqslant 3$ from a population with distribution function $F(x-\theta)$ depending on a location parameter $\theta$. If $\mathbf{D} x_{i}=\sigma^{2}<\infty$, the Pitman estimator of $\theta$ with respect to the quadratic loss,

$$
\hat{\theta}\left(x_{1}, \ldots, x_{n}\right)=\bar{x}-\mathbf{E}_{0}\left(\bar{x} \mid x_{1}-\bar{x}, \ldots, x_{n}-\bar{x}\right),
$$

is linear in observations, i.e.,

$$
\mathbf{E}_{0}\left(\bar{x} \mid x_{1}-\bar{x}, \ldots, x_{n}-\bar{x}\right)=\text { const },
$$

if and only if $F$ is Gaussian. This result known as the KLR-theorem, was proved in [2] and marked the beginning of our fruitful collaboration with Yuri Vladimirovich Linnik who would have turned now 90 .

We dedicate this paper to the memory of Linnik, the teacher of one of us and a good friend of the other.

As is well known (see, e.g., [3, Chapter 13]), the Fisher information on $\theta$ contained in an observation with distribution function $F(x-\theta)$, satisfies the inequality

$$
I \geqslant \sigma^{-2}
$$

with the equality sign again holding only for Gaussian $F$. In [1, Chapter 4] a definition of the Fisher information on a location parameter is given for an arbitrary $F$, not necessarily absolutely continuous.

* Department of Mathematics, University of Maryland, College Park, MD 20742, USA; e-mail: amk@math.umd.edu

** Department of Statistics, Pennsylvania State University, University Park, PA 16802, USA; e-mail: crr1@psu.edu 
In this paper, we consider the setup when a bivariate observation $(X, Y)$ has an informative component $X$ and ancillary $Y$ with

$$
\mathbf{P}_{\theta}\{X \leqslant x, Y \leqslant y\}=F(x-\theta, y) .
$$

The setup was first considered in [5].

Conditions for linearity of the Pitman estimator of $\theta$ are obtained as well as an inequality extending (2),

$$
I \geqslant \frac{1}{\sigma^{2}\left(1-\rho^{2}\right)}, \quad \rho=\operatorname{corr}(X, Y),
$$

the conditions for the equality sign in (3) being closely related to those for linearity of the Pitman estimator.

An open problem of interest is formulated.

A lower bound for the Fisher information. Let a bivariate random vector $(X, Y)$ have density $f(x-\theta, y)$ with $\theta \in \mathbf{R}$ as a parameter. Assuming the second moments finite and $f(x, y)$ differentiable with respect to $x$, set

$$
\begin{gathered}
\mathbf{E} X=\mu_{1}, \quad \mathbf{E} Y=\mu_{2}, \quad \mathbf{D} X=\sigma_{1}^{2}, \quad \mathbf{D} Y=\sigma_{2}^{2}, \quad \operatorname{corr}(X, Y)=\rho, \\
J=J(x-\theta, y)=\frac{\partial \ln f(x-\theta, y)}{\partial \theta}, \\
I=\mathbf{E}_{\theta} J^{2}=\iint|J(x-\theta, y)|^{2} f(x-\theta, y) d x d y=\iint|J(x, y)|^{2} f(x, y) d x d y .
\end{gathered}
$$

Theorem 1. The following inequality for the Fisher information I on $\theta$ contained in $(X, Y)$ holds:

$$
I \geqslant \frac{1}{\sigma_{1}^{2}\left(1-\rho^{2}\right)}
$$

with the equality sign if and only if

$$
f(x, y)=\exp \left\{A_{0} x^{2}+A_{1} x+B x y+C(y)\right\},
$$

where $A_{0}, A_{1}, B, C(y)$ are such that

$$
\mathbf{D} X=\sigma_{1}^{2}, \quad \operatorname{corr}(X, Y)=\rho, \quad \mathbf{D} Y<\infty .
$$

$\mathrm{P}$ r o o f. If $I=+\infty$, (4) trivially holds. Hence, one may assume $I<\infty$.

Let $\widehat{J}(x, y)=\widehat{c}_{1}\left(x-\mu_{1}\right)+\widehat{c}_{2}\left(y-\mu_{2}\right)$ be the best linear approximation of $J(x, y)$ in $L^{2}(f)$, i.e.,

$$
\widehat{J}(x, y)=\underset{c_{1}, c_{2}}{\arg \min } \iint\left[J(x, y)-c_{1}\left(x-\mu_{1}\right)-c_{2}\left(y-\mu_{2}\right)\right]^{2} f(x, y) d x d y .
$$

From $\mathbf{E}_{0}\left\{\left(X-\mu_{1}\right) J(X, Y)\right\}=-1, \mathbf{E}_{0}\left\{\left(Y-\mu_{2}\right) J(X, Y)\right\}=0$ one gets

$$
\widehat{c}_{1}=-\frac{1}{\sigma_{1}^{2}\left(1-\rho^{2}\right)}, \quad \widehat{c}_{2}=\frac{\rho}{\sigma_{1} \sigma_{2}\left(1-\rho^{2}\right)}, \quad \widehat{J}=-\frac{\left(x-\mu_{1}\right)-\rho \sigma_{1}\left(y-\mu_{2}\right) / \sigma_{2}}{\sigma_{1}^{2}\left(1-\rho^{2}\right)}
$$

and

$$
\mathbf{E}(\widehat{J}(X, Y))^{2}=\frac{1}{\sigma_{1}^{2}\left(1-\rho^{2}\right)} .
$$

Since $I=\mathbf{E}(J(X, Y))^{2} \geqslant \mathbf{E}(\widehat{J}(X, Y))^{2},(6)$ proves (4).

The equality sign in (4) holds if and only if

$$
J(x, y)=\widehat{c}_{1}\left(x-\mu_{1}\right)+\widehat{c}_{2}\left(y-\mu_{2}\right) .
$$

Integrating this relation leads to (5). Theorem 1 is proved. 
Note that the lower bound (4) does not depend on the variance of the ancillary component $Y$.

Bivariate Gaussian densities are a special case of (5) as are product densities of a Gaussian $X$ and arbitrary $Y$.

Introduce now the characteristic function

$$
\varphi(t, s)=\mathbf{E}_{0} \exp (i t X+i s Y)
$$

of $(X, Y)$ and marginal characteristic functions $\varphi_{1}(t)$ of $X$ and $\varphi_{2}(s)$ of $Y$.

Since

$$
\begin{aligned}
\iint e^{i t x+i s y} \frac{\partial \ln f(x, y)}{\partial x} f(x, y) d x d y & =-i t \varphi(t, s) \\
\iint x e^{i t x+i s y} f(x, y) d x d y & =-i \frac{\partial \varphi(t, s)}{\partial t} \\
\iint y e^{i t x+i s y} f(x, y) d x d y & =-i \frac{\partial \varphi(t, s)}{\partial s}
\end{aligned}
$$

the linearity of $J(x, y)$ in terms of the characteristic function is equivalent to

$$
t \varphi(t, s)=\widehat{c}_{1} \frac{\partial \varphi(t, s)}{\partial t}+\widehat{c}_{2} \frac{\partial \varphi(t, s)}{\partial s}+i\left(\widehat{c}_{1} \mu_{1}+\widehat{c}_{2} \mu_{2}\right) \varphi(t, s) .
$$

We will need this relation in the next section.

Linearity of the Pitman estimator. Let $\left(x_{1}, y_{1}\right), \ldots,\left(x_{n}, y_{n}\right)$ be a sample from a bivariate population with distribution function $F(x-\theta, y)$ with $\int x^{2} d F(x)<\infty$. The Pitman estimator of $\theta$ is

$$
\hat{\theta}\left(x_{1}, y_{1} ; \ldots ; x_{n}, y_{n}\right)=\bar{x}-\mathbf{E}_{0}\left(\bar{x} \mid x_{1}-\bar{x}, \ldots, x_{n}-\bar{x} ; y_{1}, \ldots, y_{n}\right) .
$$

Let us look first at the straightforward extension of the KLR-theorem.

Theorem 2. If $\hat{\theta}=\bar{x}+$ const and $n \geqslant 3$, then $x_{i}$ is Gaussian and

$$
\varphi(t, s)=\varphi_{1}(t) \varphi_{2}(s), \quad t \in \mathbf{R}, \quad|s|<\epsilon,
$$

where $\epsilon=\max \left\{s:\left|\varphi_{2}(u)\right|>0,|u|<s\right\}$. If $\varphi_{2}(s) \neq 0$, then $x_{i}, y_{i}$ are independent.

$\mathrm{P}$ r o o f. To prove Gaussianity of $x_{i}$, note that the relation

$$
\mathbf{E}_{0}\left(\bar{x} \mid x_{1}-\bar{x}, \ldots, x_{n}-\bar{x} ; y_{1}, \ldots, y_{n}\right)=\text { const }=c, \quad \text { say }
$$

implies

$$
\mathbf{E}_{0}\left(\bar{x} \mid x_{1}-\bar{x}, \ldots, x_{n}-\bar{x}\right)=c
$$

that as shown in the KLR-theorem holds for $n \geqslant 3$ if and only if $x_{i}$ is Gaussian.

The relation (9) is equivalent to

$$
\begin{aligned}
& \mathbf{E}_{0}\left(\bar{x} \exp \left\{i\left[t_{1}\left(x_{1}-\bar{x}\right)+\cdots+t_{n}\left(x_{n}-\bar{x}\right)+s_{1} y_{1}+\cdots+s_{n} y_{n}\right]\right\}\right) \\
& \quad=c \mathbf{E}_{0}\left(\exp \left\{i\left[t_{1}\left(x_{1}-\bar{x}\right)+\cdots+t_{n}\left(x_{n}-\bar{x}\right)+s_{1} y_{1}+\cdots+s_{n} y_{n}\right]\right\}\right)
\end{aligned}
$$

holding for all real $t_{1}, \ldots, t_{n}, s_{1}, \ldots, s_{n}$.

From (10) one gets

$$
\sum_{i=1}^{n}\left(\frac{\partial \varphi\left(\tau_{i}, s_{i}\right)}{\partial \tau_{i}} \prod_{j \neq i} \varphi\left(\tau_{j}, s_{j}\right)\right)=c \prod_{1}^{n} \varphi\left(\tau_{i}, s_{i}\right) \quad \text { if } \quad \sum_{1}^{n} \tau_{i}=0 .
$$

Let $\eta>0, \epsilon>0$ be such that $\varphi(t, s) \neq 0$ for $|t|<\eta,|s|<\epsilon$. On dividing both sides of (11) by $\prod_{1}^{n} \varphi\left(\tau_{i}, s_{i}\right)$ one gets

$$
\sum_{1}^{n} g\left(\tau_{i}, s_{i}\right)=c \quad \text { if } \quad \sum_{1}^{n} \tau_{i}=0, \quad|s|<\epsilon, \quad\left|\tau_{i}\right|<\eta, \quad i=1, \ldots, n,
$$


where $g(t, s)=\partial \ln \varphi(t, s) / \partial t$. On fixing $s_{1}=\cdots=s_{n}=s$, (12) becomes the Cauchy classical equation

$$
\sum_{1}^{n} g\left(\tau_{i}, s\right)=c \quad \text { if } \quad \sum_{1}^{n} \tau_{i}=0, \quad|s|<\epsilon, \quad\left|\tau_{i}\right|<\eta, \quad i=1, \ldots, n .
$$

Since $g(\tau, s)$ is continuous for $|\tau|<\eta,|s|<\epsilon$, the only solution of (13) for $n \geqslant 3$ is

$$
g(\tau, s)=A(s) \tau+B
$$

for some $A(s), B$. It is easy to see that $A(s)=A=$ const. Indeed, if $a^{\prime}=A\left(s^{\prime}\right) \neq$ $A\left(s^{\prime \prime}\right)=a^{\prime \prime}$, then by setting $\tau_{1}=\cdots=\tau_{n-1}=\tau, \tau_{n}=-(n-1) \tau ; s_{1}=\cdots=s_{n-1}=$ $s^{\prime}, \quad s_{n}=s^{\prime \prime}$ one gets from (12)

$$
(n-1) a^{\prime} \tau-(n-1) a^{\prime \prime} \tau=\text { const }
$$

which is a contradiction.

Hence, $g(t, s)=A s+B$ and

$$
\varphi(t, s)=\exp \left\{\frac{A t^{2}}{2}+B t+C(s)\right\}=\varphi_{1}(t) \varphi_{2}(s), \quad|t|<\eta, \quad|s|<\epsilon .
$$

Since $\varphi_{1}(t) \neq 0, t \in \mathbf{R},(14)$ holds for $t \in \mathbf{R},|s|<\epsilon$. If one assumes a priori that $\varphi_{2}(s) \neq 0$, then (14) holds for all real $t$ and $s$ so that $x_{i}, y_{i}$ are independent. Theorem 2 is proved.

Note in passing that if $\varphi(t, s)$ is the characteristic function of $(X, Y)$ and

$$
\varphi(t, s)=\varphi_{1}(t) \varphi_{2}(s), \quad|t|<\eta, \quad|s|<\epsilon,
$$

$X$ and $Y$ are not necessarily independent. Here is an example due to Larry Shepp. Let $\left(\varphi_{11}(t), \varphi_{12}(t)\right)$ and $\left(\varphi_{21}(s), \varphi_{22}(s)\right)$ be two pairs of characteristic functions such that

$$
\begin{aligned}
& \varphi_{11}(t)=\varphi_{12}(t)=\varphi_{1}(t), \quad|t| \leqslant 1 ; \quad \varphi_{11}(t) \neq \varphi_{12}(t), \quad|t|>1, \\
& \varphi_{21}(s)=\varphi_{22}(s)=\varphi_{2}(s), \quad|s| \leqslant 1 ; \quad \varphi_{21}(s) \neq \varphi_{22}(s), \quad|s|>1 .
\end{aligned}
$$

Such pairs exist; one may take Pólya characteristic functions (see, e.g., [4, Chapter 4]).

The function

$$
\varphi(t, s)=\frac{1}{2}\left[\varphi_{11}(t) \varphi_{21}(s)+\varphi_{12}(t) \varphi_{22}(s)\right],
$$

as a convex combination of characteristic functions, is the characteristic function of a random vector $(X, Y)$. For $|t| \leqslant 1,|s| \leqslant 1$,

$$
\varphi(t, s)=\varphi_{1}(t) \varphi_{2}(s)
$$

but the factorization does not hold for all $t$ and $s$ so that $X, Y$ are dependent.

Assume from now on that $\int y^{2} d F(x)<\infty$.

Theorem 3. If $\hat{\theta}\left(x_{1}, y_{1} ; \ldots ; x_{n}, y_{n}\right)=\bar{x}-c_{0}-C \bar{y}$ and $n \geqslant 3$, the characteristic function $\varphi(t, s)$ of $\left(x_{i}, y_{i}\right)$ satisfies relation ( 7$)$ for $|t|<\eta,|s|<\epsilon$ for some $\eta>0, \epsilon>0$. If $\varphi(t, s) \neq 0$, then it satisfies (7) for all real $t, s$ and if, moreover, the distribution of $\left(x_{i}, y_{i}\right)$ is given by a differentiable in $x$ density $f(x-\theta, y)$, then $f(x, y)$ is necessarily (5). If $\varphi(t, s)$ satisfies (7) for all real $t, s$, then the Pitman estimator of $\theta$ is linear.

$\mathrm{P} \mathrm{r}$ o o f. The first condition of Theorem 3 is equivalent to

$$
\mathbf{E}_{0}\left(\bar{x} \mid x_{1}-\bar{x}, \ldots, x_{n}-\bar{x} ; y_{1}, \ldots, y_{n}\right)=c_{0}+C \bar{y} .
$$

To simplify notation, let us assume (without loss of generality) that $\mathbf{E}_{0} X=\mathbf{E}_{0} Y=0$ in which case $c_{0}=0$. Proceeding as in the proof of Theorem 2, one gets from

$$
\mathbf{E}_{0}\left(x_{1}-\bar{x}, \ldots, x_{n}-\bar{x} ; y_{1}, \ldots, y_{n}\right)=C \bar{y}
$$

the relation (actually, an analytical equivalent of the previous relation)

$$
\sum_{i=1}^{n}\left(\frac{\partial \varphi\left(t_{i}, s_{i}\right)}{\partial t_{i}} \prod_{j \neq i} \varphi\left(t_{i}, s_{i}\right)\right) C \sum_{i=1}^{n}\left(\frac{\partial \varphi\left(t_{i}, s_{i}\right)}{\partial s_{i}} \prod_{j \neq i} \varphi\left(t_{i}, s_{i}\right)\right),
$$


whence

$$
\sum_{1}^{n}\left(\frac{\partial \ln \varphi\left(\tau_{i}, s_{i}\right)}{\partial \tau_{i}}-C \frac{\partial \ln \varphi\left(\tau_{i}, s_{i}\right)}{\partial s_{i}}\right)=0, \quad \sum_{1}^{n} \tau_{i}=0, \quad\left|\tau_{i}\right|<\eta, \quad\left|s_{i}\right|<\epsilon .
$$

As in Theorem 2, for $n \geqslant 3$ the only solution of (16) is

$$
\frac{\partial \ln \varphi(t, s)}{\partial t}-C \frac{\partial \ln \varphi(t, s)}{\partial s}=D t, \quad|t|<\eta, \quad|s|<\epsilon .
$$

On differentiating (17) in $t$ and $s$ and setting $t=s=0$, one sees that $C=\rho \sigma_{1} / \sigma_{2}$, $D=-\sigma_{1}^{2}\left(1-\rho^{2}\right)$ so that the only difference between (17) and (7) is that the former holds for $|t|<\eta,|s|<\epsilon$ while the latter holds for all real $t, s$.

If $\varphi(t, s) \neq 0,(17)$ holds for all real $t, s$. If additionally $\left(x_{i}, y_{i}\right)$ has a differentiable in $x$ density $f(x-\theta, y)$, then, as shown in Theorem $1, f(x, y)$ must be of the form (5).

Conversely, if $\varphi(t, s)$ satisfies (7) for all real $t, s$, then simple calculations show that (15) holds for all real $t_{1}, \ldots, t_{n} ; s_{1}, \ldots, s_{n}$ and hence the Pitman estimator is linear in $\bar{x}$ and $\bar{y}$.

2. Discussion and an open problem. As shown in Section 1 , if $x_{i}$ is Gaussian, $x_{i}$ and $y_{i}$ are dependent and the characteristic function of $y_{i}$ does not vanish, $\bar{x}$ can be improved (with respect to the quadratic loss) as an estimator of $\theta$ from a sample $\left(x_{1}, y_{1}\right), \ldots,\left(x_{n}, y_{n}\right)$ of size $n \geqslant 3$ from a population $F(x-\theta, y)$.

Does this principle hold true in general? In other words, if the distribution of $x_{i}$ is not necessarily Gaussian and $x_{i}, y_{i}$ are dependent, can the Pitman estimator $\bar{x}-\mathbf{E}_{0}\left(\bar{x} \mid x_{1}-\bar{x}, \ldots, x_{n}-\bar{x}\right)$ based only on the informative components $x_{1}, \ldots, x_{n}$ of a sample $\left(x_{1}, y_{1}\right), \ldots,\left(x_{n}, y_{n}\right)$ from $F(x-\theta, y)$ be always improved? If $\int x^{2} d F(x)<\infty$, the problem is whether the relation

$$
\mathbf{E}_{0}\left(\bar{x} \mid x_{1}-\bar{x}, \ldots, x_{n}-\bar{x}\right)=\mathbf{E}_{0}\left(\bar{x} \mid x_{1}-\bar{x}, \ldots, x_{n}-\bar{x} ; y_{1}, \ldots, y_{n}\right)
$$

implies independence of $x_{i}$ and $y_{i}$.

An information analog of this problem is when the Fisher information $I(X, Y)$ on $\theta$ contained in a vector $(X, Y)$ with density $f(x-\theta, y)$ equals the Fisher information $I(X)$ contained in $X$. It is easily seen that $X$ and $Y$ must be independent.

Under mild conditions, in large samples

$$
\mathbf{D} \hat{\theta}\left(x_{1}, \ldots, x_{n}\right)=\frac{1}{n I(X)}(1+o(1))
$$

while

$$
\mathbf{D} \hat{\theta}\left(x_{1}, y_{1} ; \ldots ; x_{n}, y_{n}\right)=\frac{1}{n I(X, Y)}(1+o(1))
$$

Based on this, one can expect that the answer to the above question is yes under some conditions on the sample size and the characteristic or/and density function but relations of the type (18) seem to be a challenge.

\section{REFERENCES}

1. Хьюбер П. Робастность в статистике. М.: Мир, 1984, 512 с.

2. Kagan A. M., Linnik Yu. V., Rao C. R. On a characterization of the normal law based on a property of the sample average. - Sankhyā, 1965, v. A27, p. 405-406.

3. Каган А.М., Линник Ю.В., Рао С. Р. Характеризационные задачи математической статистики. М.: Наука, 1972, 656 с.

4. Лукач E. Характеристические функции. М.: Наука, 1979, 423 с.

5. Rao C.R. Minimum variance estimation in distributions admitting ancillary statistics. - Sankhyā, 1952, v. A12, p. 53-56. 